\title{
Aspectos vivenciales en adolescentes que sobrevivieron a un cáncer de la infancia
}

\author{
Pilar Cubillos P. '; Carlos Almonte V. ${ }^{2}$; Eugenia Emparanza del S. ${ }^{3}$
}

\section{Emotional aspects in adolescents surviving cancer in infancy}

Life perceptions were studied by means of a rematic aperception tesl (MAurray) in 21 cancer survivor male and female adolescenls, who were trealed in the orcology unit of an university associated public pediatric hospital at metropolitan Santiago. Chile. Mos! polienls perceived both the world and themselves in a negative way. All of them had depressive faelings, mainly relased to confliclive family sifuations and secondarily lo death or survival as well. The importance of psychoiogicai support at the different stages of oncologic disease is emphasized, particulary in adolescent parients whose adaptation needs may beccme aculely and severily affected.

[Key words: adolescence, neoplasic, survival, emotions.)

Algunos aspectos vivenciales y caracteristicas de la sobrevivencia en niños menores de 11 años se describieron en una comunicación anterior'. La adolescencia es un período de importantes cambios corporales psicológicos y sociales, que suponen desequilibrios y ajustes. En ella se vuelven a actualizar y elaborar los conflictos y vivencias infantiles no resueltos hasta ese momento. Nuestro propósito fue indagar cómo repercuten las enfermedades oncológicas en esta etapa del desarrollo, considerando que el tiempo que ha transcurrido desde el diagnóstico de la afección tumoral es mayor que en la muestra de los niños menores recién aludida, con el objetivo de evaluar el estado de salud mental de estos jovenes y proponer -cuando resulte indicadomedidas para favorecer la superación de situaciones no resueltas, facilitando de este modo un dearrollo psicosocial armónico.

\section{Pacientes y Método}

Se consideraron sobrevivientes de cáncer infantil los jovenes que habiendo tenido la enfermedad se han mantenido libres de ella cinco o más años desde el momento de

1. Psicóloga Clínica

2. Servicio de Salud Mental, Hospital Roberto del Río.

3. Unidad de Hematología-Oncología Hospital Roberto del Río. Departamento de Pediatria. Universidad de Chile. hacer el diagnóstico o de la última recaída registrada. La inuestra estaba constituida por 2 I adolescentes - 13 mujeres-que han sido atendidos en la unidad de oncología del Servicio de Pediatría del Hospital Roberto del Río del Servicio de Salud Metropolitano Norte de Santiago. Nueve $(43 \%)$ ) 6 niñas, tenían 12 a 15 anos (adolescencia temprana); los 12 restantes -7 mujeres- mis de 15 y hasta 18 afos de edad. Los diagnósticos oncológicos eran leucemia linfoblástíca aguda (n:14, uno de ellos con recaída testicular y otro con recaída meníngea); linfoma (n:4); rabdomiosarcoma $(n: 2)$; e histiocitosis $X(n ; 1)$. La edad en el momento del diagnóstico oncologico varió entre los 4 meses y los 11 años, concentrándose la mayoria de la muestra en preescolares $(62 \%)$. Nueve de los 21 jóvenes quedaron con algún tipo de secuela física (43\%): tres con talla baja y uno de cada una de las sigujentes alteraciones: escasez de cabello, artrosis de mano e hipogonadismo, asimetria de piema, ausencia de dientes $y$ uncia inferiores, orçuidectomía y cicatriz en línea media abdominal.

El método de evaluación empleado fue similar at aplicado en un estudio previo en niños sobrevivientes de cáncer infantil $^{1}$, adaptado a la adolescencia. La evalución psicológica se orientó a determinar el funcionamiento global de los jovenes en sus aspectos cognitivos y afectivo sociales. El rendimiento escolar se estirnó del promedio de notas anuales del año anteriot, clasificándolo como bueno (5,0 a 7), regular (4 a 4,9) y deficiente (inferior a 4 o repitencia). La evaluación cognitiva se realizó en los menores de 17 años mediante la escala de inteligencia de Weschler para niños revisada (WISC-R, normas chileras): en los mayores de esa edad se empleó la versión para adultos (WAIS). Para detectar indicadores sugetentes de lesión cerebral se aplicó la prueba gestálica visomotriz de Bender Bip. En la deterninación de los aspectos vivenciales se emplearon pruebas psicologicas "proyectivas" basadas en consignas y en apercepción temática. 
Las pruebas basadas en cansignas se hicieron mediante dibujo libre de la figura humana, la casa, árbol, persona. la familia y del problema que el joven desarrolla a partir de sus propias experiencias - lo que para él adquiere mayor relevancia afectiva- o mediante la creación de un relato escrito donde los personajes son animales.

La prueba de apercepción temática de Henry A. Murray (TAT) $^{j}$ es un procedimiento clásico utilizado en el diagnóstico psicológico de adolescentes y adultos. en el cual se presentaron al sujeto 13 láminass, sobre cada una de las cuales debe desarrollar una historia. Cada lámina explora distintas situaciones a las cuales la persona se ve enfrentada en sus relaciones faniliares, sexualidad, trabajo.

Para fines de esta comunicación, a partir de las pruebas de proyección se definieron algunos criterios que permitan evaluar cualitativamente las vivencias del joven sobre si y su mundo: la percepcion del mundo se refiere a la forma en que el joven percibe el que lo rodea amenazante a amistoso: peligroso o libre de grandes peligros que amenacen su integridad). En la percepción de sí mismo pueden, a su vez, predominar sentimientos posilivos de fortaleza para enfrentar las dificultades; negativos. de inclinación a lo depresivo. con predominio de sentinientos de desvalimiento y temor; o agresivos. provocando el teiner de otros. Las ansiedades principales identifican las situaciones que la generan en mayor magnitud a los jóvenes. como por ejemplo ante la muerte, el daño fisico, ia separación, no ser querido, el castigo o la posibilidad de enloquecer. El tipo de vínculo se refiere al modo en cómo el personaje principal se relaciona con aros. pudiendo predominar las relaciones positivas, amorosas y solidarias; o las negativas, agresivas o inestables. El tipo de solución es la manera cósno el sujeto resuelve los con. flictos: puede ser positiva (permite la supervivencia de los personajes, con buena adaptación posterior): negativa (la historia termina con la destrucción de los personajes principales, o el desarrolto del cuento queda bloqueado en una situación de impotencia); o también abierta (no plantea desenlace, no se resuelve el conflicto).

A partir exclusivamente de la prueba de apercepción temática se agregaron otras dimenșiones no incluidas en la evaluación de los niños escolares, referentes al manejo de situaciones peligrosas, percepción de la situación laboral, de estudio, sexualidad, relaciones familiates, que se expresan en dicotomías, positivo o negativo. Además se indagó sobre las situaciones que el joven asocia con vivencias depresivas.

\section{Resultados}

El desarrollo cognitivo correspondió al nivel intelectual normal promedio en 13 jovenes $(62 \%$ de la muestra); еп seis $(25 \%)$ era normal lento, en uno (5\%) la inteligencia era limítrofe y en otro joven $(5 \%)$ había retardo mental. El rendimiento escolar era bueno en siete jóvenes $(33 \%)$, regular en ocho $(38 \%)$ y deficiente en seis (29\%).

De acuerdo a la clasificación multiaxial de Rutter para el diagnóstico psiquiátrico, un jo- ven se ubicaba en la variable normal (5\%). En los 20 restantes se detectaron reacciones de adaptación ( $\mathrm{n}=5 ; 3$ niñas); trastorno emocional ( $n=11 ; 8$ niñas); desorden de personalidad dependiente ( $n=3$; todos varones) y retraso mental en una niña.

La adaptación social, entendida en el modo cómo el joven se relaciona con padres, herma. nos, otros adultos y pares, era buena en seis (29\% de la muestra), regular en once $(52 \%)$ y deficiente en cuatro (19\%).

En el área cmocional, la percepción de] mundo cra amenazante para la mayoría de los jovenes. La percepción de sí mismo, predominantemente negativa, sintiéndose desvalidos. Como ansiedades principales, en número importante apareció la ansiedad de muerte, siguiendo el temor a no ser querido, y la ansiedad de separación. Los tipos de vinculo predominante fueron los negativos, principalmente los rotos y agresivos. El tipo de solución dado por los jovenes fue positivo en proporciones casi iguales que negativo o no resuelto (tabla 1).

En casi igual proporción los jóvenes se percibieron a sí mismos con y sin recursos para salir de las situaciones peligrosas. Levemente superior fue el número de jóvenes que percibieron la situación de estudio o trabajo, en conflicto: en su mayoría con sentimientos de incapacidad. Le sigue en importancia la vivencia de soledad asociada al estudio. En seis de estos jóvenes las vivencias de incapacidad están vinculadas a dificultades reales de aprendizaje o rendimiento escolar. Predominó una visión negativa del área de la sexualidad con relaciones poco gratificantes de pareja, asociado a la ansiedad de no ser querida en las mujeres y a la muerte en los varones. La percepción de las relaciones familiares fue principalmente conflictiva, con una resolución mayoritariamente negativa a las dificultades. Los sentimientos depresivos fueron asociados por los jóvenes principalmente a relaciones familiares conflictivas y a la temática de la sobrevivencia y la muerte (tabla 2 ).

En las pruebas gráficas se registraron, en estos 21 jóvenes, vivencias preferentemente depresivas en trece adolescentes (8 niñas), asociadas a sentimientos de abandono, dificultad en el contacto afectivo y fragilidad. En cuatro jóvenes (dos niñas) predominaron vive- 
Tahla 1

Dimensión afectiva evaluada a través de la prucba de apercepción temática ел 21 jóvenes sobrcvivientes de cáncer infantil

\begin{tabular}{|c|c|c|c|c|}
\hline & Mujeres & Varones & Total & $\%$ \\
\hline \multicolumn{5}{|l|}{ Percepción del mundo: } \\
\hline $\begin{array}{l}\text { Amenazante } \\
\text { Amistosa }\end{array}$ & $\begin{array}{r}12 \\
1\end{array}$ & $\begin{array}{l}7 \\
1\end{array}$ & $\begin{array}{r}19 \\
2\end{array}$ & $\begin{array}{l}90 \\
10\end{array}$ \\
\hline \multicolumn{5}{|l|}{ Percepción de sí mismo: } \\
\hline $\begin{array}{l}\text { Predominante negativa } \\
\text { Positiva }\end{array}$ & $\frac{12}{1}$ & $\begin{array}{l}7 \\
1\end{array}$ & $\begin{array}{r}19 \\
2\end{array}$ & $\begin{array}{l}90 \\
10\end{array}$ \\
\hline \multicolumn{5}{|l|}{ Ansiedades principales: } \\
\hline $\begin{array}{l}\text { Ansiedad de muerte } \\
\text { Temor al daño físico } \\
\text { Temor a no ser querido } \\
\text { Ansiedad de separación } \\
\text { Temor al castgoo } \\
\text { Temor de enloquecer }\end{array}$ & $\begin{array}{l}3 \\
3 \\
3 \\
3 \\
- \\
1\end{array}$ & $\begin{array}{l}5 \\
- \\
2 \\
i \\
1 \\
\end{array}$ & $\begin{array}{l}8 \\
3 \\
5 \\
3 \\
1 \\
1\end{array}$ & $\begin{array}{r}38 \\
14 \\
24 \\
14 \\
5 \\
5\end{array}$ \\
\hline \multicolumn{5}{|l|}{ Tipo de vínculo: } \\
\hline $\begin{array}{l}\text { Negativo } \\
\text { Positivo }\end{array}$ & $\begin{array}{l}9 \\
4\end{array}$ & $\begin{array}{l}6 \\
2\end{array}$ & $\begin{array}{r}15 \\
6\end{array}$ & $\begin{array}{l}71 \\
29\end{array}$ \\
\hline \multicolumn{5}{|l|}{ Tipo de solución: } \\
\hline $\begin{array}{l}\text { Positiva } \\
\text { Negativa } \\
\text { Sin solución }\end{array}$ & $\begin{array}{l}6 \\
2 \\
5\end{array}$ & $\begin{array}{l}2 \\
5 \\
1\end{array}$ & $\begin{array}{l}8 \\
7 \\
6\end{array}$ & $\begin{array}{l}38 \\
33 \\
29\end{array}$ \\
\hline Total & 13 & 8 & 21 & 100 \\
\hline
\end{tabular}

cias ansiosas, vinculadas a la enfermedad y la secuela física. Los cuatro jovenes restantes (tres niñas) manifestaron vivencias propias del desarrollo normal, mostrando ambivalencia con respecto a crecer $y$ ser independientes.

\section{Comentario}

La muestra estudiada se ubica en la adolescencia temprana y media. El objetivo principal de esta etapa de la vida es el fortalecimiento de la identidad, que se verá concretada en la autonomia del joven para elegir pareja, estilo de vida y trabajo $2-5$.

La adolescencia es un período de reelaboración de conflictos y vivencias infantiles y de cambios corporales, psicológicos y sociales, lo que supone importantes desequilibrios y ajustes. Los psicoanalistas describen tres procesos de duelo o pérdida en el adolescen- tc: pérdida del cuerpo infantil; duelo por dejar de ser niño dependicnte, aceptando nuevas responsabilidades; duelo por los padres de la infancia, que trata de retener buscando refugio y protección ${ }^{5}$. Por ello se espera que en los adolescentes alternen, como parte del desarrollo normal, períodos de vivencias marcadamente depresivas con olros de gran vitalidad, como asimismo sentimientos de ambivalencia en torno a crecer, que pueden reflejarse en comportamientos regresivos alternando con actitudes de madurez ${ }^{3.5}$.

Estos resultados no difïeren mayormente con los obtenidos en niños escolares sobrevivientes de cáncer' en lo que respecta a percepciones predominantemente amenazante del mundo $(90 \%$ en esta inuestra y $83 \%$ en niños menores), negativa de sí mismo $(90 \%$ y $72 \%$ ), ansicdad de muerte como principal ansiedad (38\% y $56 \%$ ) y tipo de vínculo negativo $(71 \%$ y $72 \%)$. Estos hallazgos sugieren 
Tabla 2

Dimensión afectiva evaluada a través de la apercepción temática en 21 jóvenes según la naturaleza de las situaciones propuestas

\begin{tabular}{|c|c|c|c|c|}
\hline & Mujeres & Varones & Total & $\%$ \\
\hline \multicolumn{5}{|l|}{ Manejo de situaciones peligrosas: } \\
\hline Con recursos & 8 & 2 & 10 & 48 \\
\hline Sin recursos & 5 & 6 & 11 & 52 \\
\hline \multicolumn{5}{|l|}{ Situación laboral o de estudio: } \\
\hline Sin conflicto & 5 & 4 & 9 & 43 \\
\hline En conflicto & 8 & 4 & 12 & 57 \\
\hline \multicolumn{5}{|l|}{ Percepción de la sexualidad: } \\
\hline Visión positiva & 2 & 2 & 4 & 19 \\
\hline Visión regativa & 11 & 6 & 17 & 81 \\
\hline \multicolumn{5}{|l|}{ Relaciones familiares: } \\
\hline Predominantemente positivas & 4 & 2 & 6 & 29 \\
\hline Negativas & 9 & 6 & 15 & 71 \\
\hline \multicolumn{5}{|c|}{ Sentimientos depresivos a temática de: } \\
\hline Sobrevivencia y fmuerte & 5 & 4 & 9 & 43 \\
\hline Relación famsliar conflictiva & 7 & 4 & II & 52 \\
\hline Falta de pareja & 1 & - & I & 5 \\
\hline Total & 1.3 & 8 & 21 & 100 \\
\hline
\end{tabular}

una dinámica vivencial que persiste aunque el tiempo de sobrevida supere ampliamente el de la muestra anterior. En otras palabras, persiste el temor a la recaída, el mundo es visto de un modo amenazante, por lo que hay una importante tendencia a retrotraerse, buscando refugio en los padres. Esta constelación resulta sin embargo aun más interferente en el desarrollo del adolescente por las características de este período evolutivo, donde el joven debe ejercitar su autonomía ${ }^{1,6-7}$.

De acuerdo con los resultados obtenidos, los varones tenderían a resolver los conflictos de modo negativo, con mayor frecuencia que las mujeres, percibiéndoseles con menos recursos para enfrentar situaciones de miedo y peligro. Asimismo, la ansiedad de muerte en ellos tendría una frecuencia más alta. En este sentido habría una mayor vulnerabilidad en los varones respecto a las mujeres. Estos resultados pueden ser asociados a interferencia en el desarrollo normal de sentimientos de poder que da el propio cuerpo. La actividad física y los juegos bruscos, lan propios de los muchachos, han generado mucha ansiedad en los padres de estos jóvenes, tendiendo a lo largo de su historia de sobrevivencia a ser bastante restrictivos y temerosos, pudiendo derivar en que los jóvenes se sientan en desventaja en relación al grupo de pares que no tuvo cáncer.

En esta muestra la temática de enfermedad está presente en un número importante de jóvenes, igual que en la muestra escolar, lo que indica que es un conflicto no superado que, además, se intensifica, ya que en la adolescencia se espera que se reactualicen los conflictos no resueltos de la infancia ${ }^{3,4}$.

Una proporción mayoritaria de la muestra percibe la sexualidad de modo negativo, con vínculos poco gratificantes, asociado -en las mujeres- al temor de no ser queridas y -n los varones- a la ansiedad de muerte. Tanto los varones como las mujeres que perciben la sexualidad y las relaciones de pareja de modo positivo tienen diagnóstico psiquiátrico más favorable (los cuatro con reacción de adaptación). El incremento de las vivencias depresi- 
vas registrado en estos adolescentes, coincide con lo descrito por otros autores ${ }^{8}$. En esta investigación fueron detectados principalmente en las pruebas de apercepción temática y pruebas graficicas, vinculadas al tema de la enfermedad, conflictos familiares y sentimientos de abandono. Si bien los sentimientos depresivos son considerados parte del desarrollo normal, ligados al alejamiento del rol infantil, en los jovenes estudiados hay más bicn fuertes vivencias vinculadas a la enfermedad y a sentimientos de fragilidad, que obstaculizan el ejercicio de mayor autonomía. Ser más independiente en ellos estimula vivencias de mucho abandono y sentimientos de no ser querido, ansiedades que comparativamente sufren aumento importante en relación a los resultados obtenidos en los niños escolares. Tal vez en este proceso los jovenes no siempre encuentran el apoyo adecua. do en sus padres, ya que a éstos les cuesta. en general, aceptar el crecimiento de los hijos, más aún, cuando éste ha sufrido cáncer ${ }^{15}$.

Las ansiedades persisten en el tiempo y pueden ir estructurando modos desadaptativos de sentir. Parece necesario desarrollar programas de atención integral del paciente oncológico, considerando evaluaciones psicológicas en la adolescencia de estos niños con el objeto de detectar si las tareas evolutivas están sien. do cumplidas satisfactoriamente o si son interferidas por reactivacion de situaciones conflictivas infantiles no resueltas, relacionadas con dicha enfermedad, estableciendo, cuando sea pertinente, el tratamiento que corresponda.

\section{Resumen}

En 21 adolescentes sobrevivientes de cáncer en la niñez se emplearon pruebas psicologicas de proyección con el propósito de estu- diar aspectos vivenciales. En la mayoría de ellos la percepción del mundo y de sí mismos era negativa. Se encontraron sentimientos depresivos en todos los adolescentes, predominando los relacionados con la situación familiar conflictiva, seguidos por los vinculados a la sobrevivencia o muerte. Se enfatiza la necesidad de apoyo psicológico en los distintos períodos de la evolución de las enfermedades oncológicas, principalmente en la adolescencia, donde las exigencias de adaptación propias de este período evolutivo pueden acentuare por la reactivación de conflictos infantiles no resueltos.

(Palabras clave: sobrevivencia, cáncer infantil, adolescencia, repercusión emocional.)

\section{Referencias}

1. Almonte $C$, Cubilllos P. Emparanza E: Aspectos vivenciales de njojos sobrevivientes de cáncer infantil. Rev Chil Pediatr 1995; 66: 145-149.

2. Lidz TH: La persona. su desarrollo a través del ciclo vital. Barcelona, Editorial Herder 1985: 364-436

3. Offer $D$ : Adolescent development: A normative perspective. In: Greenspan S. y Pollak G. The course of life. Vol. IV. Adolescence. Connecticut: International Universities Press 1991: 181-199.

4. Esman A: Mid-adolescence: Foundations for later psychopathology. In: Greenspan S. y Pollak G. The course of life. Vol. IV. Adolescence. Connecticul: International Universities Press I991: 283-299.

5. Krobet $M$ : El sindrome de la adolescencia normal. En: Aherastury A. y Knobel M: La adolescencia normal. Benos Aires, Ed. Paidos 1990:35-104.

6. Glazer 10: Psychiatric. Aspects of cancer in childhood and adolescence. In: Lewis $M$ : Child and adolescent psychiatry, Baltimorc, Williams and Wilkins 199!: 964-977.

7. Murray H: Test de apercepción temática, Buenos Aires, Ed. Paidos 1988:81-1 30.

8. Rona $E$, Vargas $l:$ El impacto psicológico del cáncer en el niño y adolescente. Rev Chil Pediatr 1994: 65: 48-55. 